Stochastic stability and performance estimates of packetized unconstrained model predictive control for networked control systems

by Marcus Reble, Daniel E. Quevedo, \& Frank Allgöwer

Copyright (C) 2011 IEEE.

This is an author-prepared version of the article, reprinted from the Proceedings of the 9th IEEE International Conference on Control and Automation (IEEE ICCA'11), p. 171-176.

http://ieeexplore.ieee.org/xpls/abs all.jsp?arnumber=6137929

This material is posted here with permission of the IEEE. Such permission of the IEEE does not in any way imply IEEE endorsement of any of University of Newcastle's products or services. Internal or personal use of this material is permitted. However, permission to reprint/republish this material for advertising or promotional purposes or for creating new collective works for resale or redistribution must be obtained from the IEEE by writing to pubs-permissions@ieee.org. By choosing to view this document, you agree to all provisions of the copyright laws protecting it. 


\title{
Stochastic Stability and Performance Estimates of Packetized Unconstrained Model Predictive Control for Networked Control Systems
}

\author{
Marcus Reble, Daniel E. Quevedo, and Frank Allgöwer
}

\begin{abstract}
In this work, we consider the control of discretetime nonlinear systems over unreliable packet-based communication networks subject to random packet-dropouts. In order to mitigate the influence of the packet dropouts, the controller transmits packets containing control inputs for more than one future time instant. A suitable buffering is then applied at the plant actuator side. Since we do not assume the number of consecutive packet dropouts to be bounded, we are interested in stochastic stability of the closed-loop. For the calculation of the control inputs, we propose an unconstrained model predictive control (MPC) scheme without additional terminal weighting term. This unconstrained MPC scheme shows two significant advantages. First, we do not require the knowledge of a global control Lyapunov function, but instead only a less restrictive controllability assumption, in order to guarantee stochastic stability. Second, guaranteed performance bounds on the expected infinite horizon cost of the closed-loop can be obtained.
\end{abstract}

\section{INTRODUCTION}

Due to recent developments in communication technologies, control loops are more and more frequently closed over shared packet based communication networks. The analysis and design of such systems, most commonly referred to as networked controlled systems (NCS), has attracted significant interest in research and practical applications, see e.g. [1-3] and the references therein. The use of general purpose networks has advantages, such as lower cost and simplified cabling. However, several new challenges are posed, in particular time-delays and packet dropouts are introduced by the communication network.

The use of model predictive control (MPC) with suitable buffering strategies have been proven to be effective tools in order to compensate time-delays and packet dropouts in NCS, see e.g. [4-9].

In contrast to most other networked MPC schemes, see e.g. [6-8], in the present work we do not assume that the number of consecutive packet dropouts is bounded. In contrast, we consider channels with independent and identically distributed (i.i.d.) packet dropouts. For this reason, we use a stochastic setting to investigate stability and performance of the closed-loop.

Marcus Reble and Frank Allgöwer are with the Institute for Systems Theory and Automatic Control (IST), University of Stuttgart, Germany. \{reble, allgower\}aist.uni-stuttgart.de. Research supported by the DFG Priority Programme 1305 "Control Theory of Digitally Networked Dynamical Systems" and the Cluster of Excellence in Simulation Technology (EXC 310/1) at the University of Stuttgart.

Daniel Quevedo is with the School of Electrical Engineering \& Computer Science, The University of Newcastle, NSW 2308, Australia. dquevedo@ieee.org. Research supported by Australian Research Council's Discovery Projects funding scheme (project number DP0988601).
In contrast to the similar results in [9] our setup does not require the use of a global control Lyapunov function as terminal cost function. Instead, a less restrictive controllability assumption is used, which was similarly required in addition to the global control Lyapunov function in [9] to obtain bounds on the necessary prediction horizon and packet size in order to guarantee (stochastic) stability. Furthermore, removing the terminal cost function in our MPC scheme allows the calculation of guaranteed performance bounds, which are related to results on unconstrained MPC in the deterministic setting [10-12].

The remainder of this work is organized as follows. The problem setup is described in Section II. Section III states the main assumptions used in this work and shows some preliminary results. The main results are presented in Section IV. In particular, conditions for stochastic stability and performance estimates are given. Simulation results for a numerical example are shown in Section V. The paper ends with concluding remarks in Section VI.

Notation: $\mathbb{R}^{+}$denotes the non-negative real numbers. $\mathbb{R}^{n}$ is the $n$-dimensional Euclidean space with the standard norm $|\cdot|$. Let $\mathbb{N}$ denote the natural numbers and $\mathbb{N}_{0}=\mathbb{N} \cup\{0\}$. The conditional expected value of random variable $X$ given $Y$ is denoted by $\mathbb{E}[X \mid Y]$. We write $I_{n}$ for the $n \times n$-identity matrix.

\section{Problem Setup}

In this work, we consider nonlinear discrete-time systems

$$
\begin{aligned}
x(k+1) & =f(x(k), u(k)) \\
x(0) & =x_{0}
\end{aligned}
$$

with state $x(k) \in \mathbb{R}^{n}$ and control input $u(k) \in \mathbb{U} \subseteq \mathbb{R}^{m}$.

\section{A. Networked Control Setup and Buffering}

All data transmitted between controller and actuator is sent in large time-stamped packets. However, the presence of a packet-based network may introduce packet dropouts due to transmission errors and congestion. The effect of the packet dropouts is modeled by a discrete Bernoulli process $d(k)$, $k \in \mathbb{N}_{0}$,

$$
d(k)= \begin{cases}1 & \text { packet dropout occurs at time } k, \\ 0 & \text { packet successfully transmitted at time } k .\end{cases}
$$

The random variables $d(k)$ are i.i.d. Bernoulli variables with

$$
\operatorname{Prob}(d(k)=1)=p, \quad \operatorname{Prob}(d(k)=0)=1-p,
$$

i.e. the probability of a packet dropout for each packet is given by the constant dropout-rate $0<p<1$. 
Packet Dropouts

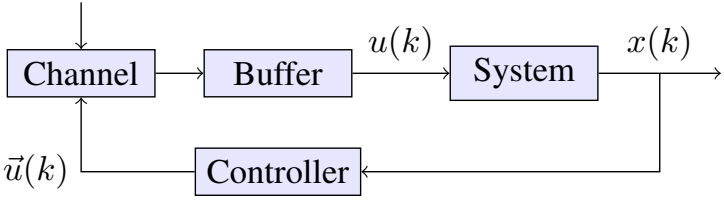

Fig. 1. Schematic of the Networked Control System.

To overcome the effects of the packet dropouts, more than one future control move is calculated and sent to the plant. At each time instant $k$, the packetized predictive controller sends a packet

$$
\vec{u}(k)=\left[u_{k \mid k}, \ldots, u_{k+N_{B}-1 \mid k}\right]^{T} \in \mathbb{U}^{N_{B}} \subseteq \mathbb{R}^{m N_{B}}
$$

to the plant, containing $N_{B}$ possible future control inputs. If the packet arrives at the buffer, the control inputs stored in $\vec{u}(k)$ are applied to the system sequentially until the next valid control packet arrives. Formally, we can describe the buffering mechanism as in [9] by using the buffer state $b(k) \in \mathbb{R}^{m N_{B}}$ and

$$
b(k)=d(k) S b(k-1)+(1-d(k)) \vec{u}(k), \quad u(k)=e_{1}^{T} b(k)
$$

in which the initial buffer state $b(0)=0$ and

$$
\begin{aligned}
S & =\left[\begin{array}{ccccc}
0 & I_{m} & 0 & \cdots & 0 \\
\vdots & \ddots & \ddots & \ddots & \vdots \\
0 & \cdots & 0 & I_{m} & 0 \\
0 & \cdots & \cdots & 0 & I_{m} \\
0 & \cdots & \cdots & \cdots & 0
\end{array}\right] \in \mathbb{R}^{m N_{B} \times m N_{B}}, \\
e_{1} & =\left[\begin{array}{ccccc}
I_{m} & 0 & \cdots & \cdots & 0
\end{array}\right] \in \mathbb{R}^{m \times m N_{B}} .
\end{aligned}
$$

In the following, we denote the successful transmission times (no packet dropouts) by

$$
\mathbb{K}=\left\{k_{0}, k_{1}, \ldots\right\} \subseteq \mathbb{N}_{0}, \quad k_{i+1}>k_{i} .
$$

Since the dropout process is Bernoulli, the time between two consecutive successful transmissions is i.i.d. with geometric distribution

$$
\operatorname{Prob}\left(k_{i+1}-k_{i}=m\right)=(1-p) p^{m-1}, \quad \forall m \in \mathbb{N} .
$$

Without loss of generality, we assume the first successful transmission to occur at $k_{0}=0$.

Using this definition, the input applied to the system can be formally written as

$$
u(k)= \begin{cases}u_{k \mid k_{i}}, & \text { for } k-k_{i}<N_{B} \\ 0, & \text { for } k-k_{i} \geq N_{B}\end{cases}
$$

in which $k_{i} \leq k<k_{i+1}$ and $k_{i}, k_{i+1} \in \mathbb{K}$. For more details, we refer to [9].

\section{B. MPC Setup}

The need for the calculation of more than one future control input renders model predictive control (MPC) a natural choice for the control design methodology. The following finite horizon cost function is minimized at each time instant $k$ for measured state $x(k)$

$$
J_{N_{p}}(x(k), \bar{u}(\cdot))=\sum_{i=0}^{N_{p}} F(\bar{x}(i ; k), \bar{u}(i))
$$

with predicted states $\bar{x}$ given by the nominal model

$$
\begin{aligned}
\bar{x}(i+1 ; k) & =f(\bar{x}(i ; k), \bar{u}(i)), \\
\bar{x}(0 ; k) & =x(k) .
\end{aligned}
$$

Here, $\mathbb{N} \ni N_{p} \geq 2$ denotes the prediction horizon. Further assumptions on the stage cost $F$ will be made in Assumption 1 in Section III. In the following, we assume that the prediction horizon is larger than or equal to the buffer size, i.e. $N_{p} \geq N_{B}$.

Note that we employ neither terminal weighting terms nor terminal state constraints in our optimization problem in contrast to the most common setups with guaranteed stability [13]. Unconstrained MPC schemes are especially attractive from a computational point of view and allow the calculation of guaranteed performance bounds $[10,11]$, see also Section IV-C.

We denote the optimal input sequence by

$$
u_{N_{p}}^{*}(\cdot ; x(k))=\arg \min _{\bar{u} \in \mathbb{U}^{N_{p}+1}} J_{N_{p}}(x(k), \bar{u}(\cdot))
$$

and the optimal value function by

$$
J_{N_{p}}^{*}(x(k))=J_{N_{p}}\left(x(x), u_{N_{p}}^{*}(\cdot ; x(k))\right) .
$$

The input values $\vec{u}(k)=u_{N_{p}}^{*}(\cdot ; x(k))$ are sent over the network in one packet and applied until a newer packet arrives. Hence, the input applied to the system is formally defined by

$$
u(k)= \begin{cases}u_{N_{p}}^{*}\left(k-k_{i} ; x\left(k_{i}\right)\right), & \text { for } k-k_{i}<N_{B} \\ 0, & \text { for } k-k_{i} \geq N_{B}\end{cases}
$$

in which $k_{i} \leq k<k_{i+1}$ and $k_{i}, k_{i+1} \in \mathbb{K}$.

\section{Preliminaries AND Assumptions}

For the derivation of the stability results, we require the following assumptions and intermediate results.

\section{A. Unconstrained MPC}

\section{Assumption 1 (Lower Bound of the Stage Cost)}

The stage cost $F: \mathbb{R}^{n} \times \mathbb{R}^{m} \rightarrow \mathbb{R}^{+}$satisfies

$$
F(x, u) \geq F(x, 0) \geq c_{F}|x|^{2} \quad \forall x \in \mathbb{R}^{n}, u \in \mathbb{R}^{m},
$$

in which $\mathbb{R}^{+} \ni c_{F}>0$.

\section{Assumption 2 (Controllability Assumption)}

For every state $x \in \mathbb{R}^{n}$ and prediction horizon $N_{p} \in \mathbb{N}$

$$
J_{N_{p}}^{*}(x) \leq B\left(N_{p}\right) F(x, 0)
$$

holds for some bounded non-decreasing function $B: \mathbb{N} \rightarrow$ $[1, \infty) \subset \mathbb{R}^{+}$with $\lim _{N_{p} \rightarrow \infty} B\left(N_{p}\right)=B_{\infty}<\infty$.

Assumption 2 is a standard assumption in unconstrained MPC [10-12,14]. Note that Condition (7) is less restrictive 
than the assumption of the knowledge of a global control Lyapunov function as required in [9]. Furthermore, assumptions about an upper bound on the optimal cost function in terms of the stage cost similar to Assumption 2 are additionally required in [9, Corollary 9].

The following lemma is a consequence of the controllability assumption and similar to previous results on unconstrained MPC in [10-12,14].

\section{Lemma 1 (Unconstrained MPC)}

Suppose that Assumptions 1 and 2 hold and that a control packet is successfully transmitted at time $k$ and applied until $k+\Delta, \Delta \leq N_{B} \leq N_{p}$, then for all $m \in\{1, \ldots, \Delta\}$

$$
\begin{aligned}
& J_{N_{p}}^{*}(x(k+m))-J_{N_{p}}^{*}(x(k)) \\
& \leq-\alpha\left(N_{p}, m\right) \sum_{j=0}^{m-1} F\left(x(k+j), u_{N_{p}}^{*}(k+j ; x(k))\right)
\end{aligned}
$$

with $\alpha\left(N_{p}, m\right)=1-\frac{B\left(N_{p}\right)^{2}}{m\left(N_{p}+1-m\right)}$.

In classical unconstrained MPC without considering packet dropouts and with fixed prediction horizon $N_{p}$ and fixed control horizon $m$, asymptotic stability of the closedloop is guaranteed if $\alpha\left(N_{p}, m\right)>0$. This can always be achieved by choosing $N_{p}$ sufficiently large because $B\left(N_{p}\right) \leq B_{\infty}$ is bounded. More precisely, $\alpha\left(N_{p}, m\right) \rightarrow 1$ for $N_{p} \rightarrow \infty$, which corresponds to recovery of infinite horizon optimality, see e.g. $[10,11]$.

Note that $\alpha\left(N_{p}, m\right) \geq \alpha\left(N_{p}, 1\right)$ for all $m \in\left\{1, \ldots, N_{p}\right\}$. Thus, applying more than one control move, e.g. due to packet dropouts, is actually beneficial with respect to the bound for nominal performance compared to the bound for classical MPC, for which only the first control move is implemented and hence $m=1$. Thus, we can use $\alpha\left(N_{p}, 1\right)$ as a kind of worst-case estimate in the following.

Under similar assumptions as in this work, it is possible to derive other estimates for $\alpha\left(N_{p}, m\right)$ than Lemma 1, e.g. following as in [11, Theorem 5.4]. Both remarks given above also hold for these formulas for $\alpha\left(N_{p}, m\right)$. Since the calculation of $\alpha$ is not our main focus, we will use the general term $\alpha\left(N_{p}, m\right)$ for the remainder of this work instead of referring to particular formulas for $\alpha$ satisfying (8).

Furthermore, we assume $\alpha\left(N_{p}, 1\right) \geq 0$ for the remainder of the present work so that the controller achieves guaranteed asymptotic stability of the closed-loop without packet dropouts.

\section{B. Open-Loop Behavior}

Since the number of possible consecutive packet dropouts is unbounded, but the buffer length is finite, the plant is left in open-loop for some time with non-zero probability. For our subsequent analysis, we need some information about the open-loop behavior of the system. Therefore, we use the following assumption, which is a variant of $[9$, Assumption 5].

\section{Assumption 3 (Open-loop Behavior)}

There exists $\gamma \in \mathbb{R}^{+}$with $1<\gamma<\frac{1}{p}$ such that

$$
F(f(x, 0), 0) \leq \gamma F(x, 0)
$$

for every $x \in \mathbb{R}^{n}$.

\section{Main Results}

\section{A. Optimal Cost At Successful Transmission Instants}

In the first step, we state results about the optimal cost function at transmission times $k_{i}$ and abbreviate $x(0)$ by $x$ and $F(x(0), u(0))$ by $F_{0}$. Without loss of generality, we consider the first two successful transmission instants $k_{0}=0$ and $k_{1}$ in the following theorem.

\section{Theorem 2}

Suppose that Assumptions 1, 2 and 3 hold. Then

$$
\mathbb{E}\left[J_{N_{p}}^{*}\left(x\left(k_{1}\right)\right) \mid x\right]-J_{N_{p}}^{*}(x) \leq-\Xi J_{N_{p}}^{*}(x)
$$

with $\Xi$ defined by

$$
\Xi=\frac{1-p^{N_{B}}}{B\left(N_{p}\right)} \bar{\alpha}-p^{N_{B}}\left(-1+\frac{(1-p) \gamma\left(B\left(N_{p}\right)-1\right)}{1-p \gamma}\right)
$$

in which $\bar{\alpha}=\frac{1-p}{1-p^{N_{B}}} \sum_{i=1}^{N_{B}} p^{i-1} \alpha\left(N_{p}, i\right)$.

Proof: See Appendix A.

Note that $\bar{\alpha} \geq \alpha\left(N_{p}, 1\right)$ because of $\alpha\left(N_{p}, 1\right) \leq \alpha\left(N_{p}, i\right)$ for all $i \in\left\{1, \ldots, N_{p}\right\}$.

By choosing the prediction horizon $N_{p}$ and the buffer size $N_{B}$ large enough, $\Xi>0$ can always be achieved because $B$ is bounded and $p<1$. More precisely, $\Xi \rightarrow \frac{1}{B_{\infty}}$ for $N_{p}, N_{B} \rightarrow \infty$. This is important because if $\Xi>0$, stochastic stability is guaranteed for all transmission instants $k_{i} \in \mathbb{K}$. Since the sequence $x\left(k_{i}\right), k_{i} \in \mathbb{K}$ forms a Markov chain, we obtain [15]

$$
\mathbb{E}\left[J_{N_{p}}^{*}\left(x\left(k_{i}\right)\right) \mid x\left(k_{0}\right)\right] \leq(1-\Xi)^{i} J_{N_{p}}^{*}\left(x\left(k_{0}\right)\right), \forall i \in \mathbb{N} .
$$

\section{B. Stochastic Stability}

Note that so far we have only considered the successful transmission instants $k_{i} \in \mathbb{K}$. Despite the fact that $k_{i+1}-k_{i}$ is not bounded, stochastic stability of the closed-loop is proven at all time instants $k \in \mathbb{N}$ in the following theorem.

\section{Theorem 3 (Stochastic Stability)}

Suppose that Assumptions 1, 2 and 3 hold and that $\Xi$ defined in Theorem 2 satisfies $\Xi>0$. Then the system is stochastically stable

$$
\mathbb{E}\left[\sum_{i=k_{0}}^{\infty}|x(i)|^{2} \mid x\left(k_{0}\right)\right]<\infty,
$$

and consequently asymptotically mean-square stable

$$
\lim _{i \rightarrow \infty} \mathbb{E}\left[|x(i)|^{2}\right]=0 .
$$

Proof: See Appendix B. 


\section{Performance Estimate}

One significant advantage of unconstrained MPC schemes without terminal weighting term is the direct attainment of guaranteed performance bounds, see e.g. $[10,11]$. In order to evaluate performance in our stochastic setup, we compare the expected value of the infinite horizon cost of the closedloop $J_{\infty}^{\mathrm{MPC}}(x)=\mathbb{E}\left[\sum_{i=0}^{\infty} F(x(i), u(i)) \mid x\right]$, obtained when applying the control law presented in this work including buffering over the lossy network, to the infinite horizon optimal cost $J_{\infty}^{*}(x)$, obtained when no packet dropouts are present. More precisely, we are looking for a suboptimality index $\mathbb{R}^{+} \ni \alpha_{P} \leq 1$ such that

$$
\alpha_{P} \mathbb{E}\left[\sum_{i=0}^{\infty} F(x(i), u(i)) \mid x\right] \leq J_{\infty}^{*}(x) .
$$

A suboptimality index $\alpha_{P}$ close to 1 corresponds to almost infinite horizon optimality.

\section{Theorem 4 (Performance Estimate)}

Suppose that Assumptions 1, 2 and 3 hold and that $\Xi$ defined in Theorem 2 satisfies $\Xi>0$. Then the performance estimate (12) is satisfied for

$$
\alpha_{P}=\frac{\alpha\left(N_{p}, 1\right)}{1+p^{N_{B}} \frac{\Psi}{\Xi}}
$$

in which $\Xi$ is defined as in Theorem 2 and

$$
\Psi=\frac{\gamma(1-p) B\left(N_{p}\right)}{1-p \gamma}+\frac{\alpha\left(N_{p}, 1\right)}{(1-p \gamma)(1-p)}-1>0 .
$$

Proof: See Appendix C.

Note that $\alpha_{P} \rightarrow \alpha\left(N_{p}, 1\right)$ for $p \rightarrow 0$, i.e. we recover the performance estimate of the classical deterministic unconstrained MPC setup if there are no packet dropouts. Furthermore, for $N_{B}, N_{p} \rightarrow \infty$ we have $\alpha_{P} \rightarrow 1$, i.e. recovery of infinite horizon optimality, as expected. Hence, Theorem 4 clearly shows the benefit from using a buffer size $N_{B}>1$ with respect to performance.

We note that an alternative performance estimate $\alpha_{P}^{\prime}$ can be directly obtained following the lines of the proof of Theorem 3 in Appendix B and using (11). However, this estimate does not recover the performance estimate of the deterministic unconstrained MPC setup without packet dropouts, i.e. $\alpha_{P} \nrightarrow \alpha\left(N_{p}, 1\right)$ for $p \rightarrow 0$.

\section{NuMERICAL EXAMPLE}

In order to be able to calculate the infinite horizon optimal cost analytically, we consider a randomly chosen unstable linear system

$$
x(k+1)=\left[\begin{array}{ll}
0.8235 & 0.3171 \\
0.6948 & 0.9502
\end{array}\right] x(k)+\left[\begin{array}{l}
0 \\
1
\end{array}\right] u(k)
$$

with poles $\lambda_{1}(A)=0.41321, \lambda_{2}(A)=1.3605$, initial condition $x_{0}=[1,1]^{T}$ and dropout rate $p=0.2$. We choose the stage cost as $F(x, u)=x^{T} x+u^{2}$ and the prediction horizon $N_{P}=10$. This allows to calculate the performance estimate $\alpha(10,1)=0.52191$ for the dropout-free case and
TABLE I

PERFormance Estimates AND Cost For Numerical EXAMPle.

\begin{tabular}{l|ccccc}
$N_{B}$ & 4 & 5 & 6 & 7 & 8 \\
\hline$\alpha_{P}$ & 0.079088 & 0.41398 & 0.49934 & 0.51736 & 0.521 \\
$\alpha_{P}^{\prime}$ & 0.019725 & 0.085079 & 0.098218 & 0.10085 & 0.10137 \\
$J_{\infty}^{\mathrm{MPC}}$ & 9.8793 & 9.8793 & 9.8793 & 9.8793 & 9.8793
\end{tabular}

the optimal infinite horizon cost $J_{\infty}^{*}=9.8793$. Stochastic stability with packet dropouts is guaranteed by Theorem 3 for buffer size $N_{B} \geq 4$. The performance estimates for different buffer sizes are given in Table I. An improvement in the guaranteed performance and recovery of the deterministic estimate for large $N_{B}$ is clearly visible. However, the actual performance of the system is already close to infinite horizon optimality, which reveals the conservativeness of both performance estimates, $\alpha(N, m)$ and $\alpha_{P}$.

\section{CONCLuSions}

In this work, we consider the stability and performance of a networked control system subject to packet dropouts. In particular, we investigate the case when the number of consecutive packet dropouts is not bounded. We propose a packetized unconstrained MPC scheme which does not require terminal cost functions or terminal constraints. In contrast to related previous work, we do not require knowledge of a global control Lyapunov function.

We derive conditions for stochastic stability of the closedloop and give a performance bound, which allows to compare the expected cost of the closed-loop with packet dropouts to the cost of the infinite horizon optimal controller when no packet dropouts occur.

\section{APPENDIX}

\section{A. Proof of Theorem 2}

Due to the definition of the expected value and the geometric distribution (2) of $k_{1}$, it directly follows that

$$
\begin{aligned}
& \mathbb{E}\left[J_{N_{p}}^{*}\left(x\left(k_{1}\right)\right) \mid x\right]=(1-p) \sum_{i=1}^{N_{B}} p^{i-1} \mathbb{E}\left[J_{N_{p}}^{*}(x(k)) \mid k=i\right] \\
& \quad+(1-p) \sum_{i=N_{B}+1}^{\infty} p^{i-1} \mathbb{E}\left[J_{N_{p}}^{*}(x(k)) \mid k=i\right]
\end{aligned}
$$

The first sum in (15) corresponds to all cases for which $k_{1} \leq N_{B}$, i.e. the next successful transmission after time instant $k_{0}$ happens within the transmitted input sequence. Thus, the buffer has never been empty. The second sum in (15) corresponds to all cases for which $k_{1}>N_{B}$, i.e. no data has been received for at least $N_{B}$ consecutive time instants. Hence, for some time the buffer state is zero and the system is running open-loop with control input $u=0$.

For the first sum in (15), we can use (8) in order to derive

$$
\begin{array}{r}
(1-p) \sum_{i=1}^{N_{B}} p^{i-1} \mathbb{E}\left[J_{N_{p}}^{*}\left(x\left(k_{1}\right)\right) \mid k_{1}=i\right] \\
\leq\left(1-p^{N_{B}}\right)\left(J_{N_{p}}^{*}(x)-\bar{\alpha} F_{0}\right) .
\end{array}
$$


The term $\left(1-p^{N_{B}}\right)$ can nicely be interpreted as the probability to have less than $N_{B}$ consecutive packet dropouts.

For the second sum in (15), note that Assumption 2 yields $J_{N_{p}}^{*}\left(x\left(k_{1}\right)\right) \leq B\left(N_{p}\right) F\left(x\left(k_{1}\right), 0\right)$, and due to Assumption 3, it is clear that $F\left(x\left(k_{1}\right), 0\right) \leq \gamma^{k_{1}-N_{B}} F\left(x\left(N_{B}\right), 0\right)$ for $k_{1}>$ $N_{B}$. Moreover, we know that $F\left(x\left(N_{B}\right), 0\right) \leq J_{N_{p}}^{*}(x)-F_{0}$. Combining the three previous inequalities gives for $k_{1}>N_{B}$

$$
J_{N_{p}}^{*}\left(x\left(k_{1}\right)\right) \leq \gamma^{k_{1}-N_{B}} B\left(N_{P}\right)\left(J_{N_{p}}^{*}(x)-F_{0}\right)
$$

Hence, we obtain for the second sum in (15)

$$
\begin{aligned}
& (1-p) \sum_{i=N_{B}+1}^{\infty} p^{i-1} \mathbb{E}\left[J_{N_{p}}^{*}\left(x\left(k_{1}\right)\right) \mid k_{1}=i\right] \\
& \stackrel{(17)}{\leq} \frac{(1-p) B\left(N_{p}\right)}{p \gamma^{N_{B}}} \sum_{i=N_{B}+1}^{\infty}(p \gamma)^{i}\left(J_{N_{p}}^{*}(x)-F_{0}\right) \\
& =\frac{(1-p) B\left(N_{p}\right)(p \gamma)^{N_{B}+1}}{p \gamma^{N_{B}}(1-p \gamma)}\left(J_{N_{p}}^{*}(x)-F_{0}\right)
\end{aligned}
$$

Plugging (16) and (18) into (15) gives

$$
\begin{aligned}
& \mathbb{E}\left[J_{N_{p}}^{*}\left(x\left(k_{1}\right)\right) \mid x\right] \\
& \leq\left(1-p^{N_{B}}+\frac{p^{N_{B}}(1-p) \gamma B\left(N_{p}\right)}{1-p \gamma}\right) J_{N_{p}}^{*}(x) \\
& \quad-\left(\left(1-p^{N_{B}}\right) \bar{\alpha}+\frac{p^{N_{B}}(1-p) \gamma B\left(N_{p}\right)}{1-p \gamma}\right) F_{0} .
\end{aligned}
$$

It directly follows that

$$
\begin{aligned}
& \mathbb{E}\left[J_{N_{p}}^{*}\left(x\left(k_{1}\right)\right) \mid x\right]-J_{N_{p}}^{*}(x) \\
& \leq\left(-p^{N_{B}}+\frac{p^{N_{B}}(1-p) \gamma B\left(N_{p}\right)}{1-p \gamma}\right) J_{N_{p}}^{*}(x) \\
& \quad-\left(\left(1-p^{N_{B}}\right) \bar{\alpha}+\frac{p^{N_{B}}(1-p) \gamma B\left(N_{p}\right)}{1-p \gamma}\right) F_{0} .
\end{aligned}
$$

Note that the term within the parentheses before $F_{0}$ is always positive. Hence, by using again $J_{N_{p}}^{*}(x) \leq B\left(N_{p}\right) F_{0}$, we obtain

$$
\begin{aligned}
& \mathbb{E}\left[J_{N_{p}}^{*}\left(x\left(k_{1}\right)\right) \mid x\right]-J_{N_{p}}^{*}(x) \\
& \leq\left(-p^{N_{B}}+\frac{p^{N_{B}}(1-p) \gamma B\left(N_{p}\right)}{1-p \gamma}\right) J_{N_{p}}^{*}(x) \\
& \quad-\left(\left(1-p^{N_{B}}\right) \bar{\alpha}+\frac{p^{N_{B}}(1-p) \gamma B\left(N_{p}\right)}{1-p \gamma}\right) \frac{J_{N_{p}}^{*}(x)}{B\left(N_{p}\right)} \\
& \leq p^{N_{B}}\left(-1+\frac{(1-p) \gamma\left(B\left(N_{p}\right)-1\right)}{1-p \gamma}\right) J_{N_{p}}^{*}(x) \\
& \quad-\left(1-p^{N_{B}}\right) \bar{\alpha} \frac{J_{N_{p}}^{*}(x)}{B\left(N_{p}\right)}
\end{aligned}
$$

which proves the claim of Theorem 2 .

\section{B. Proof of Theorem 3}

In the following, we abbreviate $x(0)$ by $x$. By using the definition of the expected value, the formula for the sum of geometric series, $p<1$, and $p \gamma<1$, we obtain

$$
\begin{aligned}
& \mathbb{E}\left[\sum_{i=k_{0}}^{k_{1}-1}|x(i)|^{2} \mid x\right] \\
& \leq(1-p) \sum_{i=1}^{\infty} p^{i-1} \mathbb{E}\left[\sum_{j=k_{0}}^{N_{B}}|x(j)|^{2} \mid k_{1}=N_{B}\right] \\
& \quad+(1-p) \sum_{i=N_{B}+1}^{\infty} p^{i-1} \mathbb{E}\left[\sum_{j=N_{B}+1}^{k_{1}-1}|x(j)|^{2} \mid k_{1}=i\right] \\
& \quad \leq \quad(1-p) \sum_{i=1}^{\infty} p^{i-1} \frac{J_{N_{p}}^{*}(x)}{c_{F}} \sum_{i=N_{B}+1}^{\infty} p_{j=N_{B}+1}^{i-1} \frac{\gamma^{j-N_{B}}}{c_{F}} F\left(x\left(N_{B}\right), 0\right) \\
& \quad+(1-p) \sum_{i}^{\infty} J_{N_{p}}^{*}(x) \\
& \quad+(1-p) \frac{J_{N_{p}}^{*}(x)}{c_{F}} \frac{\gamma}{\gamma-1} p^{N_{B}}\left(\frac{1}{1-p \gamma}-\frac{1}{1-p}\right) \\
& \leq \beta J_{N_{p}}^{*}(x)
\end{aligned}
$$

for some finite constant $0<\beta<\infty$. Similarly, one can show for all $k_{i} \in \mathbb{K}$

$$
\mathbb{E}\left[\sum_{j=k_{i}}^{k_{i+1}-1}|x(j)|^{2} \mid x\left(k_{i}\right)\right] \leq \beta J_{N_{p}}^{*}\left(x\left(k_{i}\right)\right) .
$$

Using the Markov chain property of $x\left(k_{i}\right)$ and (11) for the optimal cost function at transmission instants establishes

$$
\begin{aligned}
\mathbb{E}\left[\sum_{j=k_{0}}^{k_{i+1}-1}|x(j)|^{2} \mid x\left(k_{0}\right)\right] & \stackrel{(19)}{\leq} \beta \mathbb{E}\left[\sum_{\iota=0}^{i} J_{N_{p}}^{*}\left(x\left(k_{\iota}\right)\right) \mid x\left(k_{0}\right)\right] \\
& \stackrel{(11)}{\leq} \beta \sum_{\iota=0}^{i}(1-\Xi)^{\iota} J_{N_{p}}^{*}\left(x\left(k_{0}\right)\right) \\
& =\beta \frac{1-(1-\Xi)^{i+1}}{\Xi} J_{N_{p}}^{*}\left(x\left(k_{0}\right)\right) .
\end{aligned}
$$

Assumption 2 guarantees $J_{N_{p}}^{*}\left(x\left(k_{0}\right)\right)<\infty$, hence by taking the limit $i \rightarrow \infty$

$$
\mathbb{E}\left[\sum_{j=k_{0}}^{\infty}|x(j)|^{2} \mid x\left(k_{0}\right)\right] \leq \frac{\beta}{\Xi} J_{N_{p}}^{*}\left(x\left(k_{0}\right)\right)<\infty .
$$

This completes the proof of Theorem 3.

\section{Proof of Theorem 4}

In the following, we abbreviate $x(0)$ by $x, F(x(j), u(j))$ by $F_{j}$, and $\alpha\left(N_{p}, 1\right)$ by $\alpha$. Consider

$$
\begin{aligned}
& \mathbb{E}\left[J_{N_{p}}^{*}\left(x\left(k_{1}\right)\right)+\alpha \sum_{j=0}^{k_{1}-1} F_{j} \mid x\right] \\
& =(1-p) \sum_{i=1}^{\infty} p^{i-1} \mathbb{E}\left[J_{N_{p}}^{*}\left(x\left(k_{1}\right)\right)+\alpha \sum_{j=0}^{k_{1}-1} F_{j} \mid k_{1}=i, x\right] .
\end{aligned}
$$


Similar to the proof of Theorem 2, we split the infinite sum into parts. Due to Lemma 1, and $\alpha\left(N_{p}, 1\right) \leq \alpha\left(N_{p}, i\right)$ for all $i \in\left\{1, \ldots, N_{p}\right\}$, we obtain for the first part

$$
\begin{gathered}
(1-p) \sum_{i=1}^{N_{B}} p^{i-1} \mathbb{E}\left[J_{N_{p}}^{*}\left(x\left(k_{1}\right)\right)+\alpha \sum_{j=0}^{k_{1}-1} F_{j} \mid k_{1}=i, x\right] \\
\leq(1-p) \sum_{i=1}^{N_{B}} p^{i-1} J_{N_{p}}^{*}(x)=\left(1-p^{N_{B}}\right) J_{N_{p}}^{*}(x) .
\end{gathered}
$$

For the second part, we use Assumption 3 in order to obtain

$$
\begin{aligned}
(1-p) \sum_{i=N_{B}+1}^{\infty} p^{i-1} \mathbb{E}\left[J_{N_{p}}^{*}\left(x\left(k_{1}\right)\right)+\alpha \sum_{j=0}^{k_{1}-1} F_{j} \mid k_{1}=i, x\right] \\
\leq(1-p) \sum_{i=N_{B}+1}^{\infty} p^{i-1}\left(\gamma^{i-N_{B}} B\left(N_{p}\right) F_{N_{B}} \ldots\right. \\
\left.\ldots+\alpha \sum_{j=0}^{N_{B}-1} F_{j}+\alpha \sum_{j=N_{B}}^{i-1} \gamma^{j-N_{B}} F_{N_{B}}\right) \\
=\frac{p^{N_{B}} \gamma(1-p) B\left(N_{p}\right)}{1-p \gamma} F_{N_{B}} \\
+p^{N_{B}} \alpha \sum_{j=0}^{N_{B}-1} F_{j}+\sum_{i=N_{B}+1}^{\infty} p^{i-1} \alpha \frac{\gamma^{i-N_{B}}-1}{\gamma-1} F_{N_{B}} \\
=\frac{p^{N_{B}} \gamma(1-p) B\left(N_{p}\right)}{1-p \gamma} F_{N_{B}} \\
+p^{N_{B}} \alpha \sum_{j=0}^{N_{B}-1} F_{j}+p^{N_{B}} \frac{\alpha}{(1-p \gamma)(1-p)} F_{N_{B}} \\
\leq p^{N_{B}}(\Psi+1) J_{N_{p}}^{*}(x)
\end{aligned}
$$

for $\Psi$ defined in (14). In particular, note that $\frac{\alpha}{(1-p \gamma)(1-p)} \geq$ $\alpha$. Hence, combining the two parts of the infinite sum establishes

$\mathbb{E}\left[J_{N_{p}}^{*}\left(x\left(k_{1}\right)\right)-J_{N_{p}}^{*}(x)+\alpha \sum_{j=0}^{k_{1}-1} F_{j} \mid x\right] \leq p^{N_{B}} \Psi J_{N_{p}}^{*}(x)$

Moreover, the same relation holds for all $k_{\iota} \in \mathbb{K}$ when replacing $x$ by $x\left(k_{\iota}\right)$ and $x\left(k_{\iota}\right)$ by $x\left(k_{\iota+1}\right)$. Thus,

$$
\begin{aligned}
& \mathbb{E}\left[J_{N_{p}}^{*}\left(x\left(k_{i+1}\right)\right)-J_{N_{p}}^{*}(x)+\alpha \sum_{j=k_{0}}^{k_{i+1}-1} F_{j} \mid x\right] \\
& =\sum_{\iota=0}^{i} \mathbb{E}[\underbrace{\left.J_{N_{p}}^{*}\left(x\left(k_{\iota+1}\right)\right)-J_{N_{p}}^{*}\left(x\left(k_{\iota}\right)\right)+\alpha \sum_{j=k_{\iota}}^{k_{\iota+1}-1} F_{j} \mid x\right]}_{=\Lambda(\iota)} \\
& \stackrel{\mathrm{MC}}{=} \sum_{\iota=0}^{i} \mathbb{E}\left[\mathbb{E}\left[\Lambda(\iota) \mid x\left(k_{\iota}\right)\right] \mid x\right] \\
& \leq p^{N_{B}} \Psi \sum_{\iota=0}^{i} \mathbb{E}\left[J_{N_{p}}^{*}\left(x\left(k_{\iota}\right)\right) \mid x\right] \\
& \stackrel{(11)}{\leq} p^{N_{B}} \Psi \frac{1-(1-\Xi)^{i+1}}{\Xi} J_{N_{p}}^{*}(x) .
\end{aligned}
$$

Note that we used the Markov chain property of $x\left(k_{i}\right)$ in the step denoted by MC. Since $J_{N_{p}}^{*} \geq 0$, taking the limit of $i \rightarrow \infty$ yields $\alpha \mathbb{E}\left[\sum_{j=0}^{\infty} F_{j} \mid x\right] \leq J_{N_{p}}^{*}(x)+p^{N_{B}} \Psi \frac{1}{\Xi} J_{N_{p}}^{*}(x)$. Finally, the proof directly follows from $J_{N_{p}}^{*}(x) \leq J_{\infty}^{*}(x)$.

\section{REFERENCES}

[1] J. Baillieul and P. J. Antsaklis, "Control and communication challenges in networked real-time systems," Proc. IEEE, vol. 95, no. 1, pp. 9-28, 2007.

[2] J. P. Hespanha, P. Naghshtabrizi, and Y. Xu, "A survey of recent results in networked control systems," Proc. IEEE, vol. 95, no. 1, pp. 138$162,2007$.

[3] A. S. Matveev and A. V. Savkin, Estimation and control over communication networks. Boston, MA, USA: Birkhäuser, 2009.

[4] G. P. Liu, J. X. Mu, D. Rees, and S. C. Chai, "Design and stability analysis of networked control systems with random communication time delay using the modified MPC," Int. J. Control, vol. 79, no. 4, pp. 288-297, 2006.

[5] P. L. Tang and C. W. De Silva, "Stability validation of a constrained model predictive networked control system with future input buffering," Int. J. Control, vol. 80, no. 12, pp. 1954-1970, 2007.

[6] R. Findeisen and P. Varutti, "Stabilizing nonlinear predictive control over nondeterministic communication networks," in Nonlinear Model Predictive Control: Towards New Challenging Applications, ser. Lecture Notes in Control and Information Sciences, L. Magni, D. Raimondo, and F. Allgöwer, Eds. Springer Verlag, 2009, pp. 167-179.

[7] G. Pin and T. Parisini, "Stabilization of networked control systems by nonlinear model predictive control: a set invariance approach," in Nonlinear Model Predictive Control: Towards New Challenging Applications, ser. Lecture Notes in Control and Information Sciences, L. Magni, D. Raimondo, and F. Allgöwer, Eds. Springer Verlag, 2009, pp. 195-204.

[8] L. Grüne, J. Pannek, and K. Worthmann, "A networked unconstrained nonlinear MPC scheme," in Proc. Europ. Contr. Conf., 2009, pp. 371376.

[9] D. E. Quevedo and D. Nešić, "On stochastic stability of packetized predictive control of non-linear systems over erasure channels," in Proc. 8th IFAC Symposium on Nonlinear Control Systems (NOLCOS 2010), Bologna, Italy, 2010, pp. 557-562.

[10] L. Grüne, "Analysis and design of unconstrained nonlinear MPC schemes for finite and infinite dimensional systems," SIAM Journal on Control and Optimization, vol. 48, no. 2, pp. 1206-1228, 2009.

[11] L. Grüne, J. Pannek, M. Seehafer, and K. Worthmann, "Analysis of unconstrained nonlinear MPC schemes with time-varying control horizon," SIAM Journal on Control and Optimization, vol. 48, no. 8 , pp. 4938-4962, 2010.

[12] M. Reble and F. Allgöwer, "Unconstrained nonlinear model predictive control and suboptimality estimates for continuous-time systems," in Proc. 18th IFAC World Congress, Milan, Italy, 2011, pp. 6733-6738.

[13] D. Q. Mayne, J. B. Rawlings, C. V. Rao, and P. O. M. Scokaert, "Constrained model predictive control: stability and optimality," $\mathrm{Au}$ tomatica, vol. 26, no. 6, pp. 789-814, 2000.

[14] G. Grimm, M. J. Messina, S. E. Tuna, and A. R. Teel, "Model predictive control: for want of a local control Lyapunov function, all is not lost," IEEE Trans. Autom. Control, vol. 50, no. 5, pp. 546-558, 2005.

[15] H. J. Kushner, Introduction to Stochastic Control. New York, NY, USA: Holt, Rinehart and Winston, 1971. 